\title{
Article \\ Intelligent Combined Neural Network and Kernel Principal Component Analysis Tool for Engine Health Monitoring Purposes
}

\author{
Maria Grazia De Giorgi *(D, Luciano Strafella (D, Nicola Menga and Antonio Ficarella (D) \\ Department of Engineering for Innovation, The University of Salento, 73100 Lecce, Italy; \\ luciano.strafella@unisalento.it (L.S.); nicola.menga@unisalento.it (N.M.); antonio.ficarella@unisalento.it (A.F.) \\ * Correspondence: mariagrazia.degiorgi@unisalento.it
}

check for updates

Citation: De Giorgi, M.G.; Strafella, L.; Menga, N.; Ficarella, A. Intelligent Combined Neural Network and Kernel Principal Component Analysis Tool for Engine Health Monitoring Purposes. Aerospace 2022, 9, 118. https://doi.org/10.3390/ aerospace 9030118

Academic Editors: Spiros Pantelakis, Andreas Strohmayer and

Liberata Guadagno

Received: 15 January 2022

Accepted: 21 February 2022

Published: 24 February 2022

Publisher's Note: MDPI stays neutral with regard to jurisdictional claims in published maps and institutional affiliations.

Copyright: (c) 2022 by the authors. Licensee MDPI, Basel, Switzerland. This article is an open access article distributed under the terms and conditions of the Creative Commons Attribution (CC BY) license (https:/ / creativecommons.org/licenses/by/ $4.0 /)$.

\begin{abstract}
An efficient maintenance plan is an important aspect for aeronautical companies to increase flight safety and decrease costs. Modern technologies are widely used in the Engine Health Monitoring (EHM) discipline to develop intelligent tools capable of monitoring the health status of engines. In this work, Artificial Neural Networks (ANNs) and in-detail Feed-Forward Neural Networks (FFNNs) were exploited in addition to a Kernel Principal Component Analysis (KPCA) to design an intelligent diagnostic tool capable of predicting the Performance Parameters (PPs) of the main components used as their health index. For this purpose, appropriate datasets containing information about degraded engines were generated using the Gas Turbine Simulation Program (GSP). Finally, the original datasets and the reduced datasets obtained after the application of KPCA to the original datasets were both used in the training and testing process of neural networks, and results were compared. The goal was to obtain a reliable intelligent tool useful for diagnostic purposes. The study showed that the degraded component detection and estimation of its performance achieved by using the hybrid KPCA-FFNNs were predicted with accurate and reliable performance, as demonstrated through detailed quantitative confusion matrix analysis.
\end{abstract}

Keywords: engine health monitoring; diagnostics; artificial neural network; principal component analysis; artificial intelligence

\section{Introduction}

Diagnostics is nowadays a key concept used to ensure flight safety and cost reduction. Engine Health Monitoring is a discipline that takes care of keeping the state of health of the engine under control. Such a strategy leads to a health-based maintenance plan that stops the engine only when necessary, unlike a maintenance plan based on flight hours, as used in the past [1]. Degradation is a phenomenon caused by various factors that have a more or less serious impact on engine performance. Degrading phenomena that impact gas turbines (industrial and aeronautical) are numerous, such as fouling, erosion, abrasion, corrosion, increase in blade tip clearance, object ingestion (foreign object damage or domestic object damage), and exhaustive explanations are available in $[2,3]$. The effect on engine performance varies according to the component affected by the degradation and the phenomenon that has occurred. Compressors and turbines are the most impacted components by fouling and erosion, and their degradation is most investigated in the literature. Fouling is predominant in compressors, and it is the most prevalent degradation problem [4]. Fouling results in a decrease in the flow of both capacity and efficiency if it occurs on compressors and turbines. Erosion has the same effect of fouling on the compressor, while in the case of turbine erosion, efficiency continues to decrease but flow capacity increases [5]. For example, as reported in [6], a compressor fouling on an industrial gas turbine leads to a drop of $5 \%$ in mass flow and of $1.8 \%$ in compressor efficiency, respectively, in turn resulting in a $7 \%$ reduction in output power and $2.5 \%$ increase in 
heat rate. Meanwhile, [5] reported an increase in flow capacity of $4 \%$ and a decrease in efficiency of $2 \%$ due to turbine erosion. Erosion damage on compressors and turbines blades can be reduced by applying a coating. The results reported in [7], where a study has been conducted on the protective capabilities of various coatings against erosion and corrosion on compressor blades, show how coatings obtained by physical vapor deposition, especially the (Ti, Al)N coatings, provide the best results both in erosion and corrosion protection. In works [8,9], an Ingested Debris Monitoring System (IDMS) and an Engine Distress Monitoring System (EDMS) are used to monitor the debris present in the airflow ingested by a jet engine. The environmental impact on engine degradation is studied in $[10,11]$, where the effect of sand and dust and the effect of volcanic ash on engines are illustrated, respectively. In the former, two experiments on the effect of sand ingestion are illustrated, one considering an "accelerate" approach, in which a big amount of sand is injected into the engine inlet in a short time, and the other considering an amount of sand approaching the one typical of a real case. In each case, experiments result in a decrease in thrust and an increase in specific fuel consumption. In the second, fan blade erosion due to volcanic ash is investigated using computational fluid dynamics tools considering two different approaches, one in which the erosion rate is considered constant and the other in which it was updated over time. In both cases, the results show an increase in material removed when moving from the midspan toward the tip. Furthermore, on the suction side, for regions close to the hub the highest material removal is located around the leading edge, this then moves toward the midchord for the outer midspan sections while the pressure side experiments with a lower amount of erosion, which is focused near the midchord of the tip. EHM techniques widely exploit computer algorithms for their purposes, which are efficient diagnostic tools based on information from sensors installed in the engine itself, such as temperatures or pressures. In [12], Remaining Useful Life (RUL) is predicted by means of genetic algorithms, decision trees, and fuzzy logic to create a hybrid model. Findings show good results in RUL prediction of a few time series. The authors underline how the performances start to be good around the 150th cycle and state that the result is expected because, as stated in [13], RUL prediction becomes accurate after some cycles have already been performed by the units, allowing the degradation to accumulate. In [14], MultiGene Genetic Programming (MGGP) is used to obtain a relationship between Exhaust Gas Temperature (EGT) and other variables characterizing an engine, and in the same work, Nonlinear AutoRegressive with eXogenous inputs neural network are exploited for a one-step-ahead prediction tool. The authors also investigated the effect of the number and the nature of the parameters used in the input for MGGP and ANN algorithms, showing the network's ability to deliver accurate results even with fewer input parameters. Two Nonlinear AutoRegressive neural networks are used in [15] for specific fuel consumption estimation. In particular, the first network was used to predict fuel flow rate, compressor rotational speed, turbine inlet temperature, and compressor pressure ratio, providing in input ambient conditions, Mach number, and Power Lever Angle (PLA), while the second network was used to predict the target performance parameter, i.e., specific fuel consumption, providing in input the parameters predicted by the first network plus ambient condition and Mach number. Predictions were performed with the engine in healthy condition and simulating a compressor degradation. In each case, results showed good behavior of the developed system, with an adaption process in the case of compressor degradation. In [16], Auto-Associative Neural Networks (AANNs) are used to implement a nonlinear Principal Component Analysis (PCA) for early warning of impending gas turbine failure, and results are compared with the one obtained by applying a standard linear PCA. Results show that although the training of an AANN model takes more time than it takes for a linear PCA, the former is more suitable to be applied in nonlinear systems. PCA was exploited for feature extraction purposes in [17] to develop a PCA-ANN fault classifier for diagnostic purposes. Results show very good performance compared with other algorithms, at the cost of higher computation times. Finally, neural networks are used in $[18,19]$ to distinguish between different degradation conditions, i.e., the compressor 
fouling and the turbine erosion, with good results. In particular, in [18], results show component degradation and health condition identification EGT seems more accurate than fuel flow rate to be used as the input of the neural network, and as also evident in [19], results are better when the networks are trained with more information related to the engine (at least for the cases analyzed in these works).

Hence, Artificial Neural Networks are among the data-driven methods most widely used and very promising in the field of component degradation and fault diagnosis thanks to the solid ability in learning non-linear changes between a set of inputs and a set of outputs, as in the case of an aeronautical engine.

In this work, an FFNN is used to predict the PPs of some selected components constituting a turboshaft engine, i.e., polytropic efficiency and flow capacity for the compressor and high-pressure turbine, polytropic efficiency for the low-pressure turbine, pneumatic and combustion efficiencies for the burner, and mechanical efficiencies for shafts (as called "spools" in the present article). In addition to compressor and turbines, in our work, we also include burner and shafts transmissions, for which the degradation diagnostic is less investigated in the literature. GSP software was used to generate datasets containing information about degraded engines to be used to train neural networks. The just-mentioned datasets are noise-free. The datasets were obtained performing a series of steady-state simulations, each characterized by a different degradation condition (fault type) and severity. The degradation was implemented by changing the value of the PPs in the GSP model used for simulations. The degraded values of the PPs were obtained by an adequate equation between a maximum and a minimum value. Finally, the KPCA technique was used to reduce the amount of data to be supplied to the neural networks. In the past, several studies have used Principal Component Analysis (PCA). However, this technique has a reduced performance when applied to strongly non-linear problems, such as in the case of health monitoring of an aircraft engine. For this reason, Kernel-PCA (KPCA) has been used in this paper to overcome the limitations of conventional PCA to linear problems. ANNs were trained both with the original dataset (as obtained from simulations) and with the reduced datasets (after application of KPCA) and prediction results were compared. Compressor degradation was stimulated by a decrease in polytropic efficiency and flow capacity. Turbine degradation was considered a decrease in polytropic efficiency with both an increase and a decrease in flow capacity. For burner and shafts, degradation was simulated by a decrease in the efficiencies that characterize them.

\section{Engine Block Model}

The software exploited to model the engine used in this work was GSP, which has a drag and drop interface capable of simulating engine performance in various conditions, both in steady-state and transient conditions. More details about GSP are available in [20].

\section{Modeled Engine: Features and Design Point}

The engine used in this work was a double-spool, lightweight turboshaft designed for helicopter application, derived from the PW200 Pratt \& Whitney Canada family. A high-pressure spool connects a single-stage centrifugal compressor to a single-stage highpressure turbine (HPT), while the low-pressure spool is used to power the rotor (with a nominal speed of $6000 \mathrm{rpm}$ ) through a low-pressure turbine (LPT). The model validation was performed in standard sea-level static condition, i.e., temperature $=288.25 \mathrm{~K}$, pressure $=1.013 \mathrm{bar}$, altitude $=0$, and Mach $=0$. The airflow rate was changed until power indicated on the engine reference database was obtained. The engine block model is shown in Figure 1, while Table 1 reports some engine parameters in design conditions. The indicated stations in Figure 1 are the following: 1 -engine inlet; 2 -compressor inlet; 3-compressor outlet; 4-HPT inlet; 45-LPT inlet; 46-LPT outlet; 9-exhaust gases. 


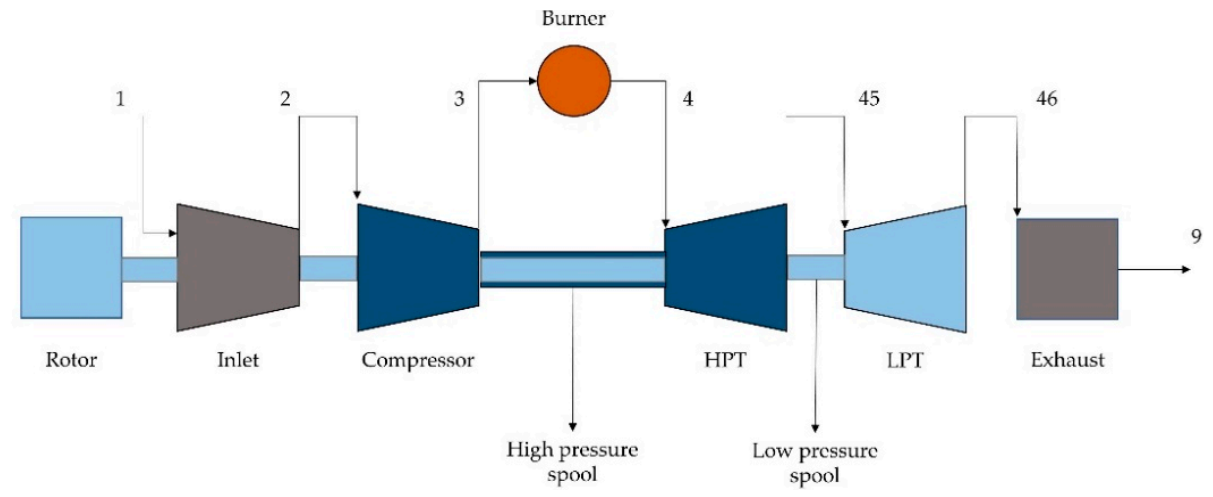

Figure 1. Block model of the used engine.

Table 1. Engine parameters in design point.

\begin{tabular}{cc}
\hline Parameter & Value \\
\hline Rotor shaft power $\left(\mathrm{P}_{\mathrm{r}}\right)$ & $295 \mathrm{KW}$ \\
Intake air mass flow rate $\left(\mathrm{w}_{1}\right)$ & $2.05 \mathrm{Kg} / \mathrm{s}$ \\
Compressor pressure ratio $\left(\beta_{\mathrm{c}}\right)$ & 7.17 \\
Compressor polytropic efficiency $\left(\eta_{\mathrm{c}}\right)$ & 0.82 \\
HPT polytropic efficiency $\left(\eta_{\mathrm{HPT}}\right)$ & 0.88 \\
LPT polytropic efficiency $\left(\eta_{\mathrm{LPT}}\right)$ & 0.88 \\
Burner pneumatic efficiency $\left(\eta_{\mathrm{b}, \mathrm{p}}\right)$ & 0.96 \\
Burner combustion efficiency $\left(\eta_{\mathrm{b}, \mathrm{c}}\right)$ & 0.985 \\
Fuel mass flow rate $\left(\mathrm{w}_{\mathrm{f}}\right)$ & $0.0314 \mathrm{Kg} / \mathrm{s}$ \\
High-pressure shaft speed $\left(\omega_{\mathrm{HPS}}\right)$ & $40,891 \mathrm{rpm}$ \\
Low-pressure shaft speed $\left(\omega_{\mathrm{LPS}}\right)$ & $6000 \mathrm{rpm}$ \\
\hline
\end{tabular}

\section{Dataset Generation: Simulation of Degraded Engines}

The degradation scenarios simulated in this work were compressor degradation, HPT degradation, LPT degradation, burner degradation, shafts degradation, and multicomponent degradation, i.e., a simultaneous degradation of the components previously analyzed individually. Table 2 describes the variables used as PPs of each component together with the related values in healthy condition at the design point and the range of percentage variation from the healthy condition. The dataset used to train ANNs was generated with GSP through a series of steady-state simulations performed on the working points described in Table 3, which were taken from the mission profiles present in [21]. For each degradation condition, 4500 steady-state simulations were performed (750 times each working point). In detail, the results of 1500 of 4500 simulations were used as testing cases, while the remaining 3000 were used as training cases. However, some simulations were discharged because they represent extreme conditions in which the software fails to converge. Each simulation is characterized by a different level of degradation, i.e., a different percentage variation of the PPs with respect to a healthy condition. The degraded PPs were obtained using Equation (1), which generates a random value of the PPs between a maximum and a minimum:

$$
p p_{d}=\left[1-\left(1-\frac{M I N}{M A X}\right) R A N^{3}\right] M A X
$$

where $p p_{d}$ is the degraded value of the PP, MIN and MAX are the lower and the upper limits, respectively, and $R A N$ is a randomly generated number between 0 and 1 . Equation (1) was used for each PP. Input and output variables for neural network training were chosen between the ones present in the datasets obtained from GSP simulations. In detail, as the goal of the work was to predict the PP of the degraded component (i.e., for each degradation condition), the neural networks must be trained on the correlation between the PPs and some characteristics variables of the engine operating status. 
Table 2. Performance Parameters of each degraded component. $\eta_{\mathrm{c}}$ : compressor polytropic efficiency; $\mathrm{f}_{\mathrm{c}}$ : compressor flow capacity; $\eta_{\mathrm{HPT}}$ : HPT polytropic efficiency; $\mathrm{f}_{\mathrm{HPT}}$ : HPT flow capacity; $\eta_{\mathrm{LPT}}$ : LPT polytropic efficiency; $\eta_{\mathrm{b}, \mathrm{p}}$ : burner pneumatic efficiency; $\eta_{\mathrm{b}, \mathrm{c}}:$ burner combustion efficiency; $\eta_{\mathrm{HPS}}$ : highpressure shaft mechanical efficiency; $\eta_{\text {LPS }}$ : low-pressure shaft mechanical efficiency.

\begin{tabular}{cccc}
\hline Component & $\begin{array}{c}\text { Degraded Performance } \\
\text { Parameters }\end{array}$ & Healthy Condition & $\begin{array}{c}\text { Range of Percentage } \\
\text { Variation }\end{array}$ \\
\hline \multirow{2}{*}{ Compressor } & $\eta_{\mathrm{c}}$ & 0.82 & $-20-0 \%$ \\
& $\mathrm{f}_{\mathrm{c}}$ & 1 & $-20-0 \%$ \\
\hline \multirow{2}{*}{$\mathrm{HPT}$} & $\eta_{\mathrm{HPT}}$ & 0.88 & $-20-0 \%$ \\
& $\mathrm{f}_{\mathrm{HPT}}$ & 1 & $-10-+10 \%$ \\
\hline \multirow{2}{*}{$\mathrm{LPT}$} & $\eta_{\mathrm{LPT}}$ & 0.88 & $-20-0 \%$ \\
\hline \multirow{2}{*}{ Burner } & $\eta_{\mathrm{b}, \mathrm{p}}$ & 0.96 & $-20-0 \%$ \\
& $\eta_{\mathrm{b}, \mathrm{c}}$ & 0.985 & $-20-0 \%$ \\
\hline \multirow{2}{*}{ Shaft transmissions } & $\eta_{\mathrm{HPS}}$ & 0.99 & $-20-0 \%$ \\
\hline \multirow{2}{*}{} & $\eta_{\mathrm{LPS}}$ & 0.99 & $-20-0 \%$ \\
\hline
\end{tabular}

Table 3. Working point simulated in steady-state simulations.

\begin{tabular}{cccc}
\hline Working Point Name & Speed $(\mathbf{m} / \mathbf{s})$ & Altitude $(\mathbf{m})$ & Power $(\mathbf{K W})$ \\
\hline START-A & 30.6 & 0 & 48 \\
START-B & 0 & 1150 & 173 \\
MAXPOW-A & 30.6 & 0 & 291 \\
MAXPOW-B & 3.96 & 1154 & 273 \\
MAXDUR-A & 30.6 & 492 & 169 \\
MAXDUR-B & 0 & 2550 & 176 \\
\hline
\end{tabular}

The variables that were chosen as input for ANNs are summarized in Table 4. These variables represent the input matrix for each neural network used while the output variables change from case to case, representing the PPs to be predicted for each degradation case, i.e., the PPs associated with the degraded component. The KPCA technique was used in this paper to reduce the input matrix dimension. Such a strategy could lead to a more efficient EHM system because the amount of data to be processed is lower compared to the case in which a data reduction technique is not used. In detail, for each of the six degradation cases, two neural networks were trained, one with the dataset consisting of the simulation results (called original dataset) and the other with the dataset obtained by applying KPCA to the original dataset (called reduced dataset).

Table 4. Variables used as input for the developed EHM system. Tot. T: Total Temperature; Tot. P.: Total Pressure.

\begin{tabular}{cc}
\hline & Inputs \\
\hline Flight Mach $(\mathrm{M})$ & Tot. T. at HPT inlet $\left(\mathrm{TT}_{4}\right)$ \\
Ambient Tot. P. $\left(\mathrm{TP}_{\mathrm{a}}\right)$ & Tot. P. at HPT inlet $\left(\mathrm{TP}_{4}\right)$ \\
Ambient Tot. T. $\left(\mathrm{TT}_{\mathrm{a}}\right)$ & Tot. T. at HPT outlet $\left(\mathrm{TT}_{45}\right)$ \\
Power lever angle $(\mathrm{PLA})$ & Tot. P. at HPT outlet $\left(\mathrm{TP}_{45}\right)$ \\
Tot. T. at comp. inlet $\left(\mathrm{TT}_{2}\right)$ & Tot. T. at LPT outlet $\left(\mathrm{TT}_{46}\right)$ \\
Tot. P. at comp. inlet $\left(\mathrm{TP}_{2}\right)$ & Tot. P. at LPT outlet $\left(\mathrm{TP}_{46}\right)$ \\
Tot. T. at comp. outlet $\left(\mathrm{TT}_{3}\right)$ & LPT torque $\left(\mathrm{T}_{\mathrm{LPT}}\right)$ \\
Tot. P. at comp. outlet $\left(\mathrm{TP}_{3}\right)$ & Overall Pressure Ratio $(\mathrm{OPR})$ \\
$\omega_{\text {HPS }}$ & Fuel mass flow rate/power lever angle $(\rho)$ \\
\hline
\end{tabular}

\section{EHM System: Combined KPCA + FFNN Algorithm}

In this section, there is a brief description of how KPCA and FFNN work. Subsequently, the setup that was used is described. 


\subsection{Feed_Forward Neural Network: Principle of Operation and Setup}

ANNs are artificial intelligence techniques designed to mimic the capacity of the human nervous system to learn from experience. The FFNN is the network type used in this work. FFNNs are constituted by layers (input layer, output layer, and hidden layers) and neurons, connected by links each having their own "weight". The input layer is the one in which information given to the network enters and the number of its neurons is equal to the number of variables given in the input. The output layer is the one from which the network predictions exit, and the number of its neurons is equal to the number of variables to be predicted. There are hidden layers between input and output layers, each constituted by a certain number of hidden neurons. The network performance depends on the number of hidden layers and hidden neurons. Equation (2) represents the output of hidden and output neurons, intended as the sum of the information (each characterized by its connection weight) deriving from the previous neurons and a bias.

$$
y=\sum_{i=1}^{n} W_{i} X_{i}+b
$$

where $y$ denotes the neuron output, $W_{i}$ is the weight of the connection between the neuron and the $i$-th previous neuron sending it information, $X_{i}$ is the information obtained from the $i$-th previous neuron, $n$ is the number of previous neurons, and $b$ is the bias. Finally, an activation function is applied to normalize the results of Equation (2). To train a neural network means providing the network with a dataset constituted by input variables and related output variables and letting the network compute the values of weights and biases in order to predict the output variables starting from the input ones. In Table 5, the neural networks trained with the original datasets are described, while in Table 6, the neural networks trained with the reduced datasets are described. Each neural network used has only one hidden layer. In the training process, a maximum of 5000 epochs was set. Figure 2a shows a typical structure of an FFNN. Figure $2 b$ instead reports the neural networks implemented with MATLAB for each case analyzed, i.e., for each degradation condition both with original and reduced datasets and having a structure like the one shown in Figure 2a.

Table 5. Characteristics of neural networks trained with original datasets.

\begin{tabular}{cccc}
\hline Degradation Condition & Input Neurons & Hidden Neurons & Output Neurons \\
\hline Compressor degradation & 18 & 26 & 2 \\
HPT degradation & 18 & 26 & 2 \\
LPT degradation & 18 & 26 & 1 \\
Burner degradation & 18 & 26 & 2 \\
Shaft transmissions degradation & 18 & 26 & 2 \\
Multi-component degradation & 18 & 34 & 9 \\
\hline
\end{tabular}

Table 6. Characteristics of neural networks with reduced datasets.

\begin{tabular}{cccc}
\hline Degradation Condition & Input Neurons & Hidden Neurons & Output Neurons \\
\hline Compressor degradation & 9 & 26 & 2 \\
HPT degradation & 9 & 26 & 2 \\
LPT degradation & 9 & 26 & 1 \\
Burner degradation & 9 & 26 & 2 \\
Shaft transmissions degradation & 9 & 26 & 2 \\
Multi-component degradation & 13 & 34 & 9 \\
\hline
\end{tabular}




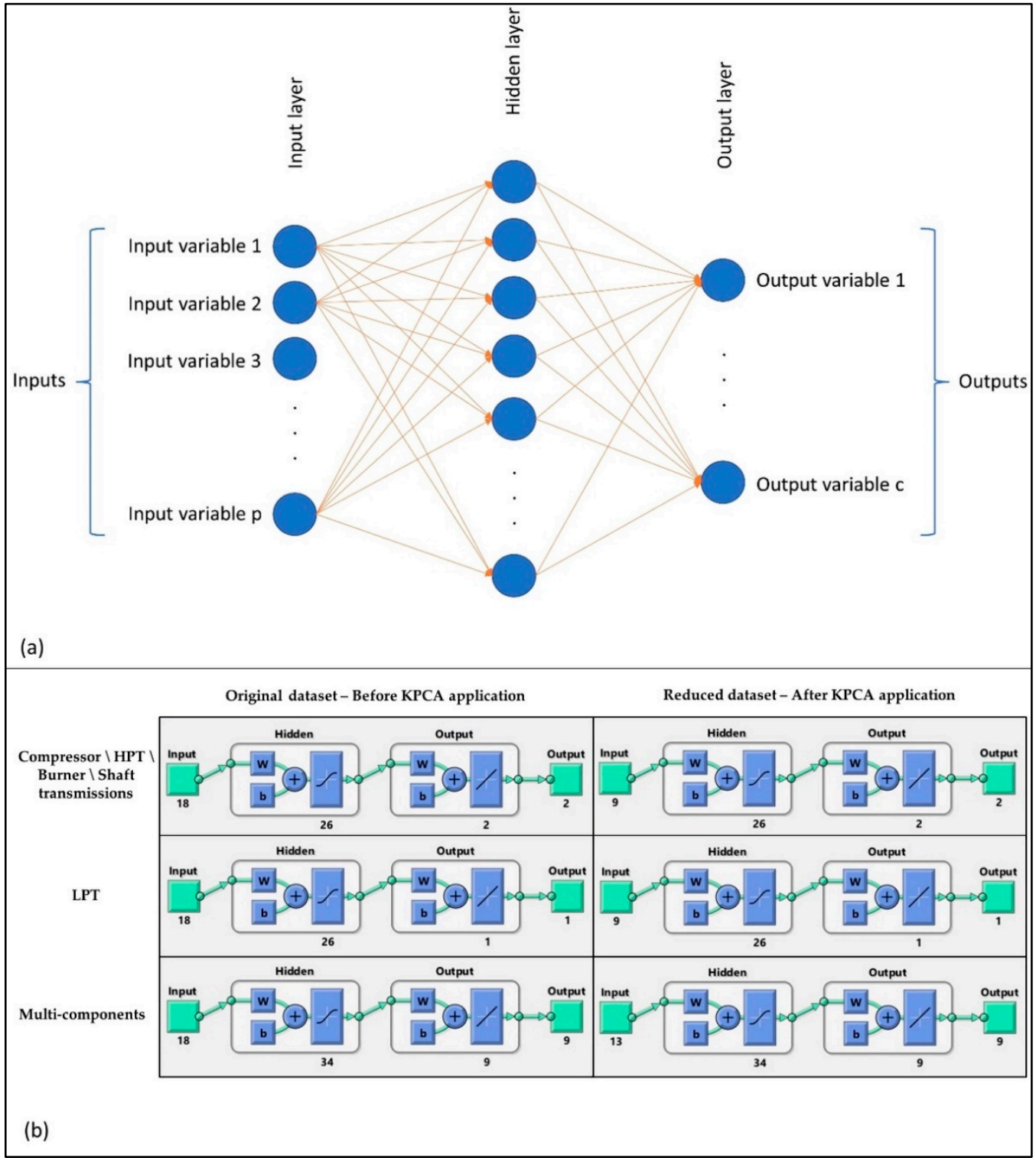

Figure 2. A typical structure of an FFNN (a) and neural networks used in this work (b), whose structure is based on the one represented in the top.

\subsection{KPCA: A Data Reduction Technique}

Principal Component Analysis is a data reduction technique used to reduce the dimension of datasets to be supplied to appropriate algorithms. However, PCA is more suitable for application in linear systems. For nonlinear systems, such as an aircraft engine, better performance is obtainable by KPCA, which combines the linear PCA with the Kernel method. The linear PCA creates a new dataset containing the same number of variables (called principal components) and the same amount of information [22] of the dataset of which is applied (original dataset). The new variables are numerically different from the ones located in the original dataset but depend on them. The principal components are ordered in the new dataset from the one containing the most information of the original dataset to the one containing the least. The strategy of the PCA is to discard the principal components having the least part of the information (the latest); in this manner, the new dataset is smaller than the original one, but carries most of the information contained in it. 
The first step of PCA is the standardization of the original dataset. The new dataset $X_{\text {new }}$ (formed by the principal components) is obtained from Equation (3):

$$
X_{\text {new }}=X_{\text {std }} Q
$$

where $X_{s t d}$ is the original dataset after standardization and $Q$ is the matrix formed by the sorted eigenvectors of the covariance matrix of $X_{s t d}$. The KPCA approach is to perform a mapping of the input data into a feature space using nonlinear mapping and subsequently perform a linear PCA in the feature space [23].

\section{Results and Discussion}

Here, the results obtained from the network predictions, both with and without the use of KPCA, are exposed. Figure 3 shows the comparison between the network predictions and the target values of the PPs obtained from GSP simulations for the first five degradation cases, i.e., for the single-component degradation cases. In more detail, the just-mentioned results are related to the predictions obtained from ANNs trained with the original datasets. Figure 4 reports the related percentage errors. From these first two figures, it is evident how the EHM system developed using only FFNNs shows very good results as $\mathrm{R}^{2}$ (coefficient of determination) values are higher than 0.95 ; in particular, the lowest $\mathrm{R}^{2}$ is equal to 0.9582 , related to $f_{c}$. The high quality of the prediction is confirmed by the low values of the percentage errors reported in Figure 4. For the compressor case, for which the related PPs are $\eta_{c}$ and $f_{c}$, a peak in error for a well-defined set of points is present at the working conditions MAXPOW-B, i.e., flight in altitude at high power. Percentage errors remain, however, below $6 \%$ for these two PPs. The remaining PPs are predicted with percentage errors all below $0.6 \%$. In Figures 5 and 6 instead, the predictions of the same variables by the KPCA + FFNN EHM system are reported. In this case, the results are slightly worse than the previous ones but are still acceptable. The lowest $R^{2}$ is again related to $f_{C}$ and is equal to 0.9869 ; therefore, the prediction of $f_{C}$ is even better with the application of KPCA. Peak errors related to the MAXPOW-B working condition are still evident and remain below $6 \%$. The remaining predictions have shown a percentage error below $0.5 \%$. Figures 7-10 are related to the prediction of the PPs for the multi-component degradation case and report in more detail the comparison between predictions of FFNN without KPCA reduction and targets, the percentage errors for the predictions of FFNN without KPCA reduction, the comparison between the prediction of FFNN with KPCA reduction and targets, and the percentage errors for the predictions of FFNN with KPCA reduction, respectively. In this case, results are worse than those obtained in the prediction of the PPs in the single-component scenario. This is an unsurprising result because, in this case, the neural networks must predict the nine variables instead of two or only one, as in the case of LPT degradation. However, results for the prediction of FFNN without KPCA remain above 0.92 with an $R^{2}$ value related to $f_{c}$ equal to 0.9211 , which remains the lowest value. The percentage errors related to this case all remain above $5 \%$, except for $f_{c}$, for which errors remain below $30 \%$. However, it is important to note from Figure $8 \mathrm{~b}$ that only some points are characterized by a high percentage error. Figures 9 and 10 show the performance for the prediction of the PPs from the KPCA + FFNN system in the multi-component degradation case. Additionally in this case, the lower performance in $\mathrm{f}_{\mathrm{c}}$ prediction is confirmed with an $R^{2}$ value equal to 0.9211 . Percentage errors of the case do not exceed $4 \%$, except for the $f_{c}$ prediction, whose percentage errors remain below 20\%. However, also in this case, only some points are characterized by a higher percentage error. 


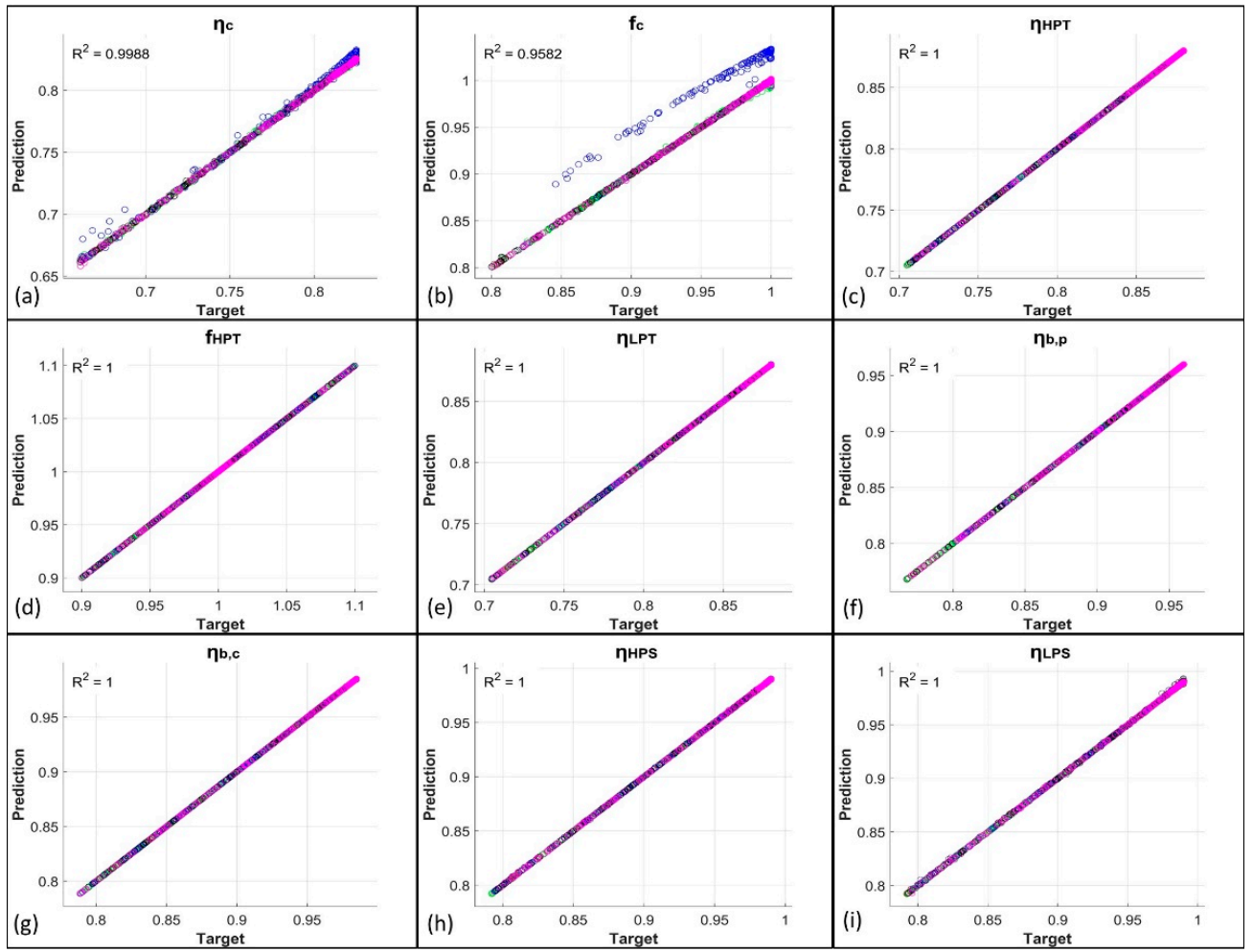

Figure 3. (a-i) Comparison between FFNN predictions and targets about the PPs related to the first five degradation conditions. Grey: START-A; brown: START-B; green: MAXPOW-A; blue: MAXPOWB; black: MAXDUR-A; magenta: MAXDUR-B.

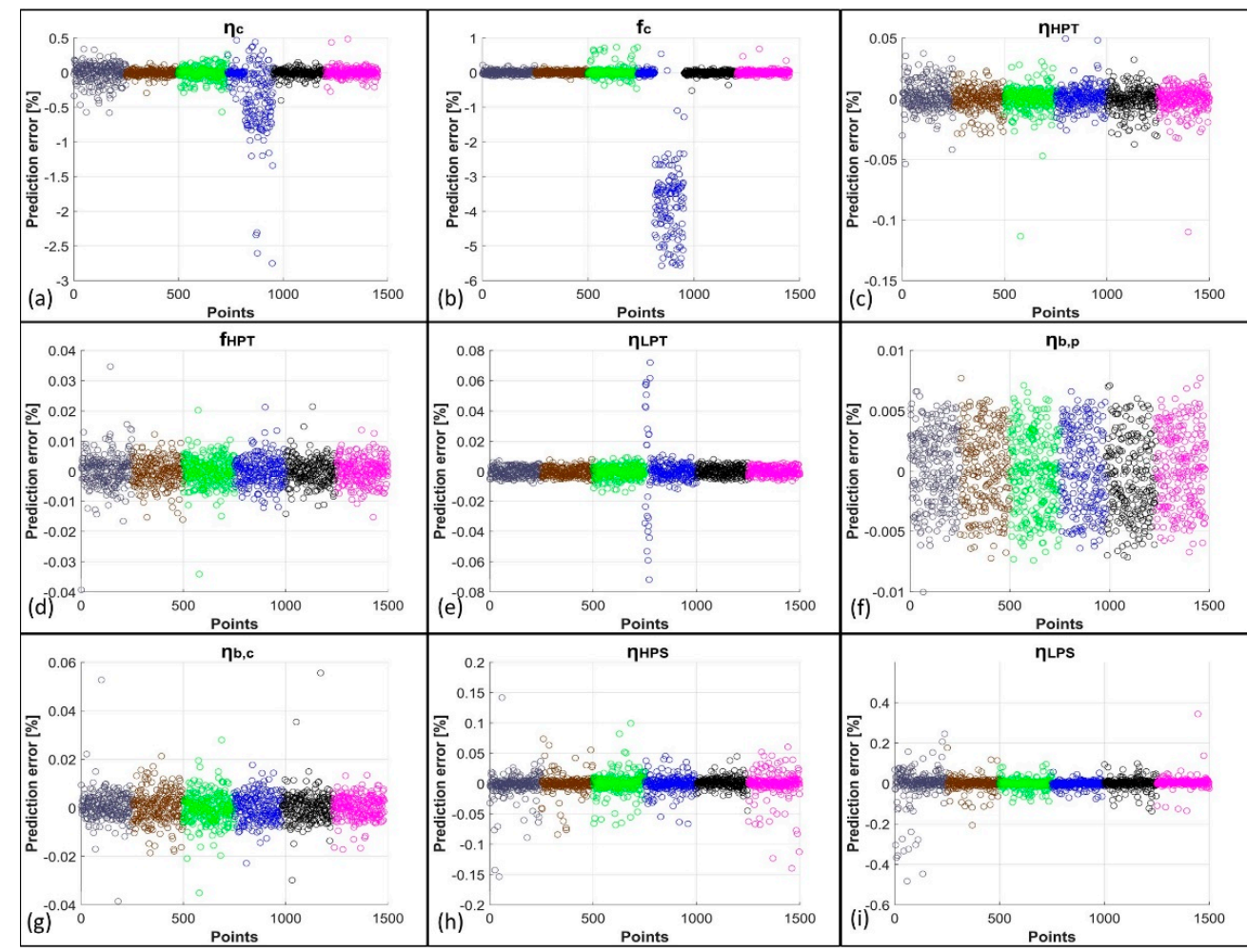

Figure 4. (a-i) Percentage errors between FFNN predictions and targets about the PPs related to the first five degradation conditions. Grey: START-A; brown: START-B; green: MAXPOW-A; blue: MAXPOW-B; black: MAXDUR-A; magenta: MAXDUR-B. 


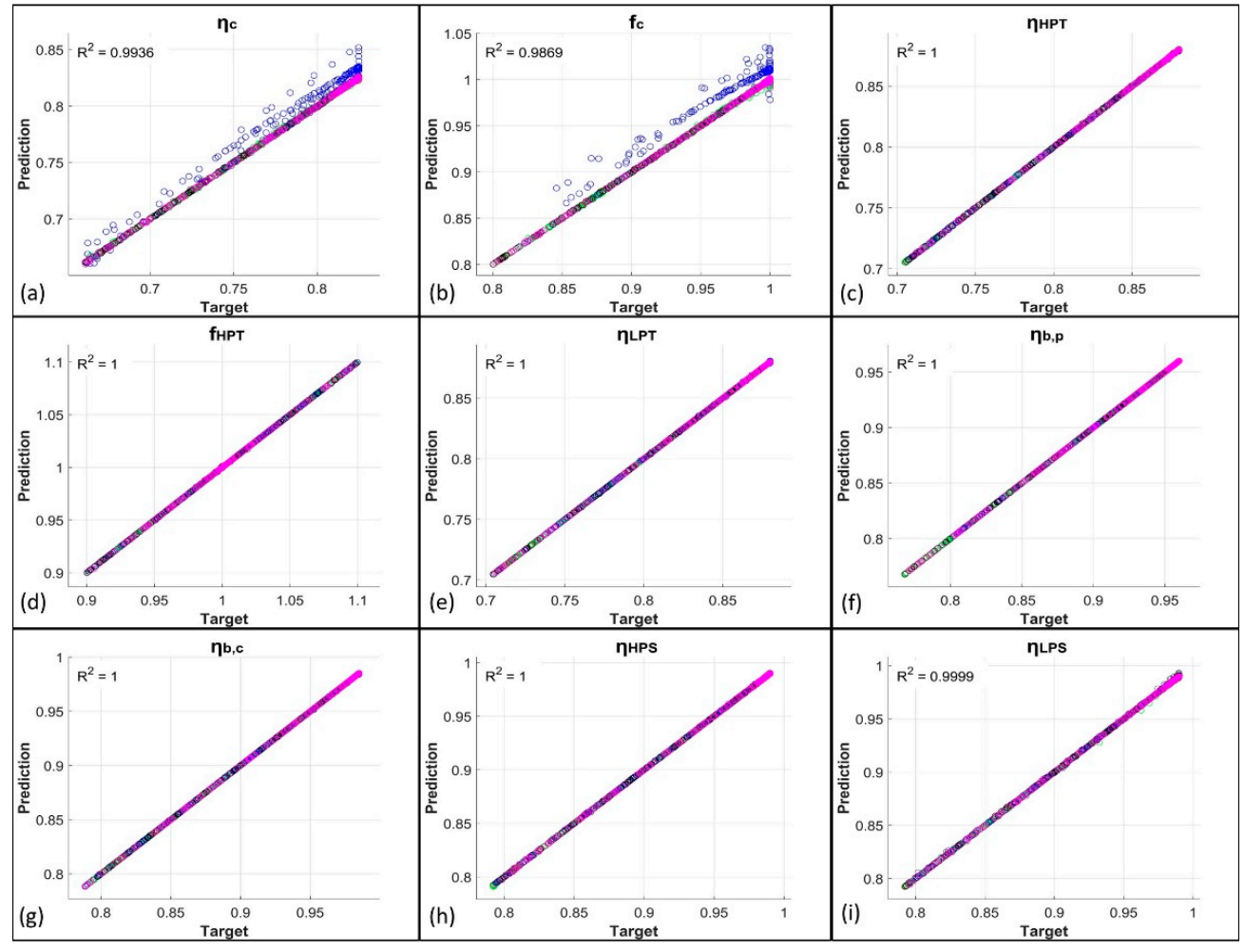

Figure 5. (a-i) Comparison between KPCA + FFNN predictions and targets about the PPs related to the first five degradation conditions. Grey: START-A; brown: START-B; green: MAXPOW-A; blue: MAXPOW-B; black: MAXDUR-A; magenta: MAXDUR-B.

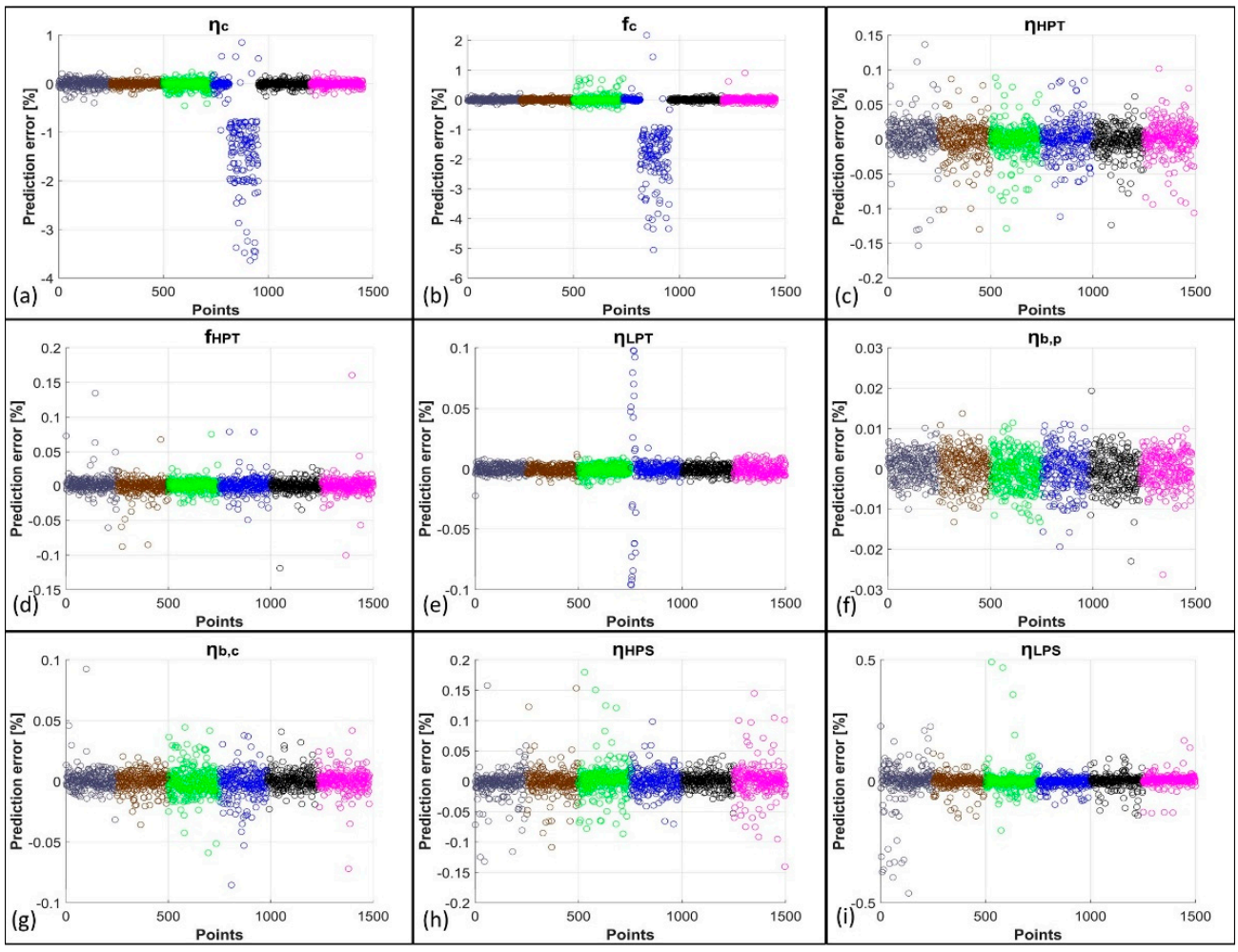

Figure 6. (a-i) Percentage errors between KPCA + FFNN predictions and targets about the PPs related to the first five degradation conditions. Grey: START-A; brown: START-B; green: MAXPOWA; blue: MAXPOW-B; black: MAXDUR-A; magenta: MAXDUR-B. 


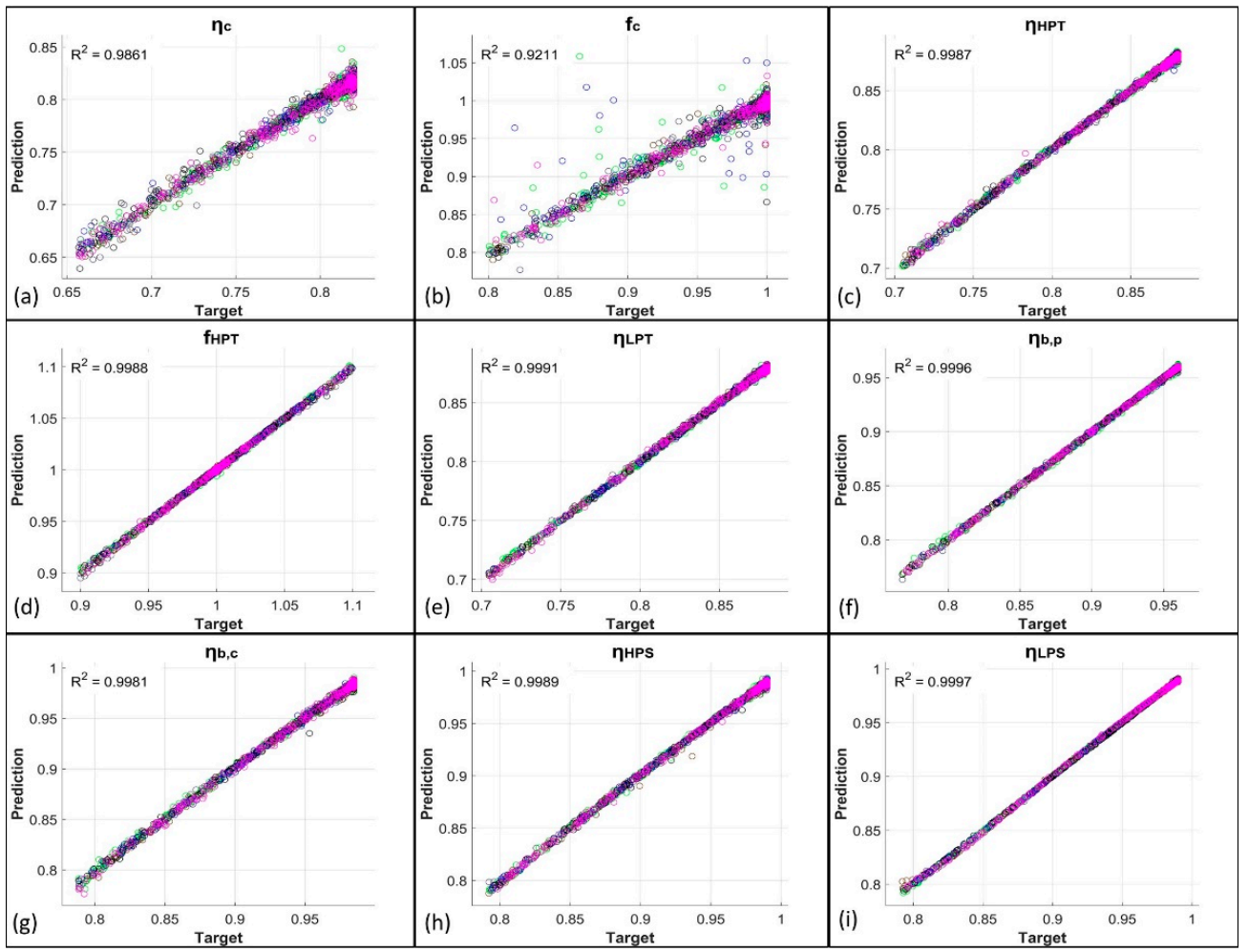

Figure 7. (a-i) Comparison between FFNN predictions and targets about the PPs related to the multi-component degradation condition. Grey: START-A; brown: START-B; green: MAXPOW-A; blue: MAXPOW-B; black: MAXDUR-A; magenta: MAXDUR-B.

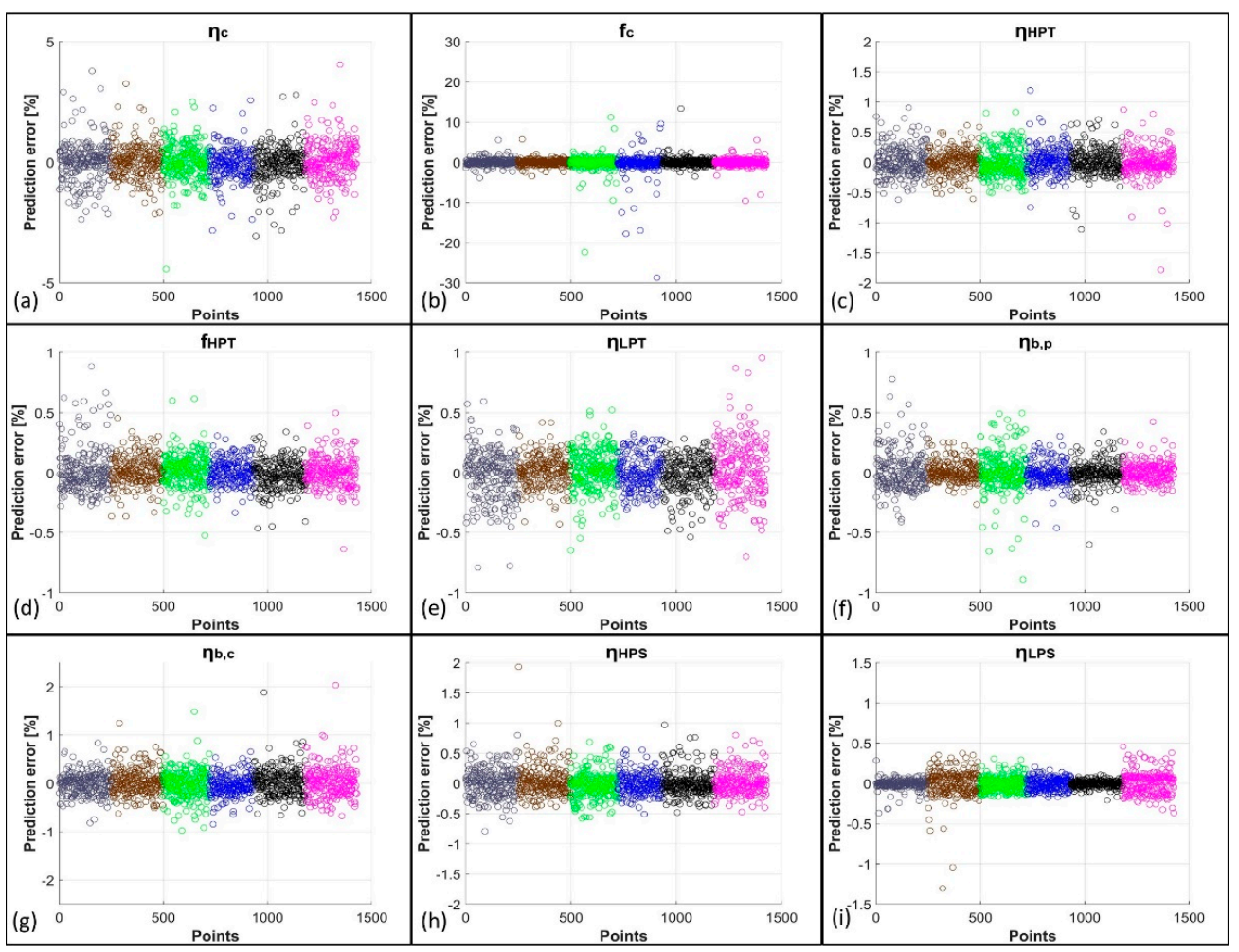

Figure 8. (a-i) Percentage errors between FFNN predictions and targets about the PPs related to the multi-component degradation condition. Grey: START-A; brown: START-B; green: MAXPOW-A; blue: MAXPOW-B; black: MAXDUR-A; magenta: MAXDUR-B. 


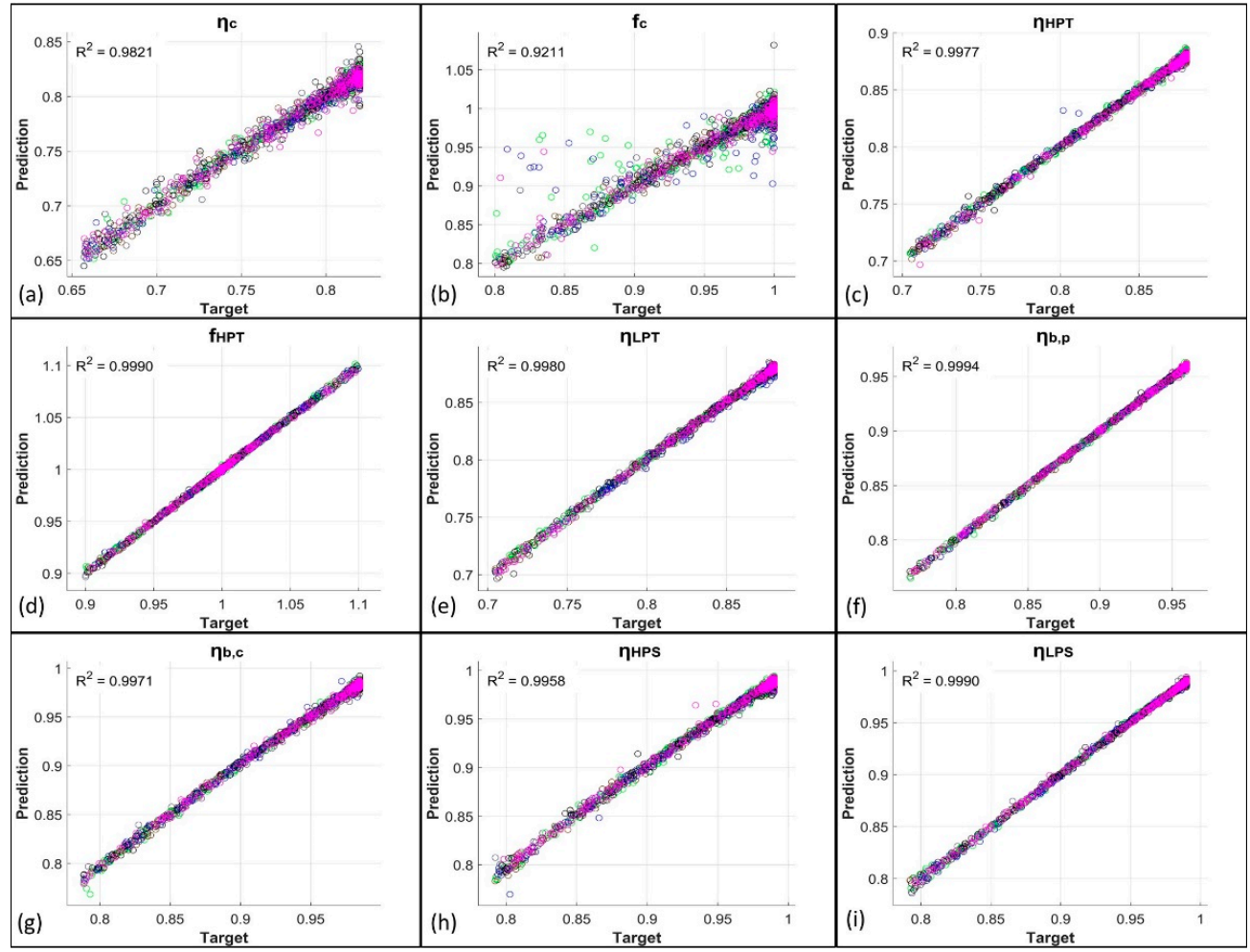

Figure 9. (a-i) Comparison between KPCA + FFNN predictions and targets about the PPs related to the multi-component degradation condition. Grey: START-A; brown: START-B; green: MAXPOW-A; blue: MAXPOW-B; black: MAXDUR-A; magenta: MAXDUR-B.

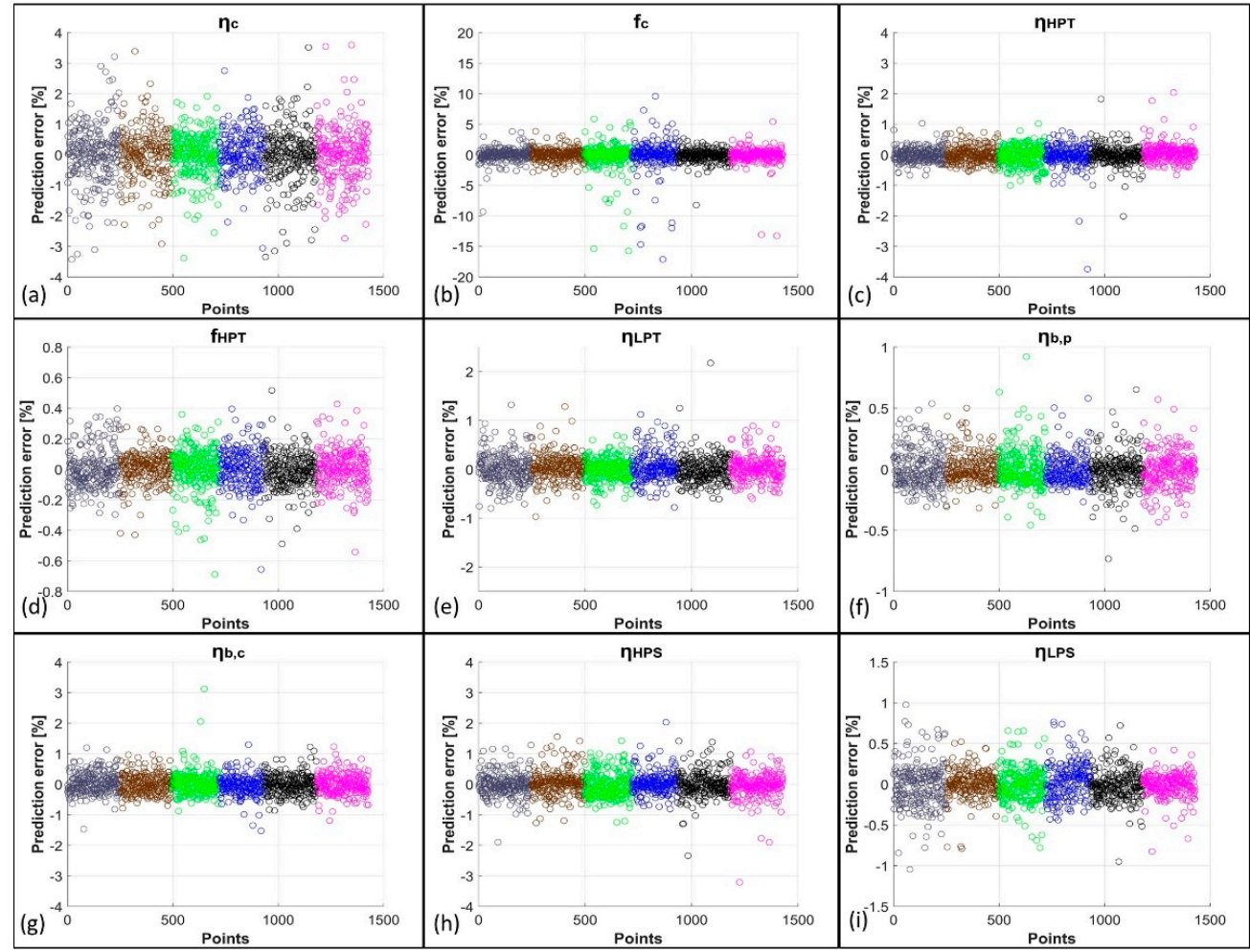

Figure 10. (a-i) Percentage errors between KPCA + FFNN predictions and targets about the PPs related to the multi-component degradation condition. Grey: START-A; brown: START-B; green: MAXPOW-A; blue: MAXPOW-B; black: MAXDUR-A; magenta: MAXDUR-B. 
The performance of the implemented system in the classification of the degraded component was evaluated. For both single-component and multi-component degradation cases, a damage class was associated with each observation based on the percentage variation of PPs with respect to the healthy condition. The classification was carried out both for the actual PPs, obtained using Equation (1) (and used to carry out the simulations), and for the PPs predicted by the machine learning system. Confusion matrices were used to establish the accuracy of the prediction method to identify the degradation status of the different engine components. Table 7 shows the damage classes for the different components based on the two PPs that were used for the compressor $\left(\eta_{c}, f_{c}\right)$, HPT ( $\eta_{H P T}$, $\left.f_{\text {HPT }}\right)$, burner $\left(\eta_{b, p}, \eta_{b, c}\right)$, and shaft transmissions $\left(\eta_{\text {HPS }}, \eta_{L P S}\right)$, while Table 8 shows the classes for LPT, for which only one PP $\left(\eta_{\mathrm{LPT}}\right)$ was considered.

Table 7. Degradation classes used for compressor, HPT, burner, and shaft transmissions.

\begin{tabular}{|c|c|c|c|c|c|}
\hline & & \multicolumn{4}{|c|}{ PP1 } \\
\hline & & PP1 $<5 \%$ & $5 \%<\mathrm{PP} 1<10 \%$ & $10 \%<P P 1<15 \%$ & $15 \%<P P 1$ \\
\hline \multirow{4}{*}{ PP2 } & $\mathrm{PP} 2<5 \%$ & 1 & 2 & 3 & 4 \\
\hline & $5 \%<\mathrm{PP} 2<10 \%$ & 2 & 3 & 4 & 5 \\
\hline & $10 \%<\mathrm{PP} 2<15 \%$ & 3 & 4 & 5 & 6 \\
\hline & $15 \%<\mathrm{PP} 2$ & 4 & 5 & 6 & 7 \\
\hline
\end{tabular}

Table 8. Damage classes used for LPT.

\begin{tabular}{cccc}
\hline PP1 $<$ 5\% & $\mathbf{5 \%}<\mathbf{P P 1}<\mathbf{1 0} \%$ & $\mathbf{P P 1} \%<\mathrm{PP1}<\mathbf{1 5 \%}$ & $\mathbf{1 5 \%}<\mathrm{PP1}$ \\
\hline 1 & 2 & 3 & 4 \\
\hline
\end{tabular}

The percentages refer to the variation with respect to a healthy condition. For example, considering the compressor, if one of the two associated PPs $\left(\eta_{c}\right.$ and $f_{c}$ ) has a percentage variation with respect to the healthy case of $6 \%$ (column 2 in Table 7 ) and the other has one of $3 \%$ (row 1 in Table 7 ), the associated damage class is 2 . The confusion matrices for the different components are shown in Figures 11-15. Regarding the compressor, the application of KPCA both in single-component and multi-component degradation cases leads to a slight drop in performance that is still acceptable. The difference between singlecomponent and multi-component cases instead is more marked, passing from $96.7 \%$ to $88.7 \%$ and from $96.4 \%$ to $87.6 \%$ of well-classified results for FFNN and KPCA + FFNN methods, respectively. For HPT, LPT, burner, and shaft transmissions, results have a slight difference both in single and multi-component degradation and both using FFNN and KPCA + FFNN methods. Lower classification performance results are reported from the prediction of the burner's PPs in multi-component degradation using KPCA + FFNN, equal to $96.3 \%$. Best results are obtained for LPT, for which there is a perfect classification in the single-component degradation case, both with FFNN and KPCA + FFNN. Based on these findings, further development of the present work would be achieved by the use of multiple single-output ANNs; in this way, it will be possible to better optimize the ANNs' settings to reduce the prediction errors in the case of multi-component degradation. Hence, it will be useful to combine the ANNs with KPCA to reduce the computational time, which increases switching from the multi-outputs single ANN approach to the multiple single-output ANNs. 


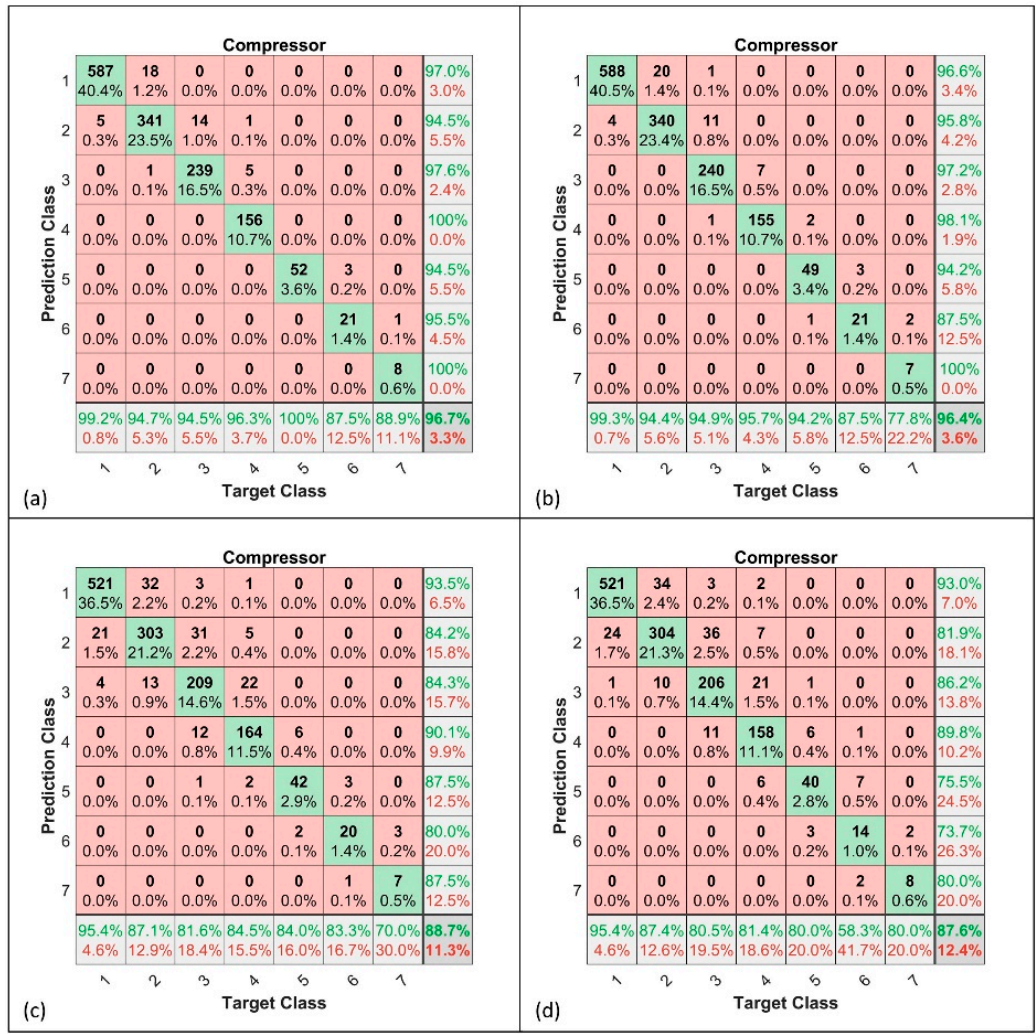

Figure 11. Confusion matrix for compressor. (a): Single-component FFNN; (b): single-component KPCA + FFNN; (c): multi-component FFNN; (d): multi-component KPCA + FFNN.

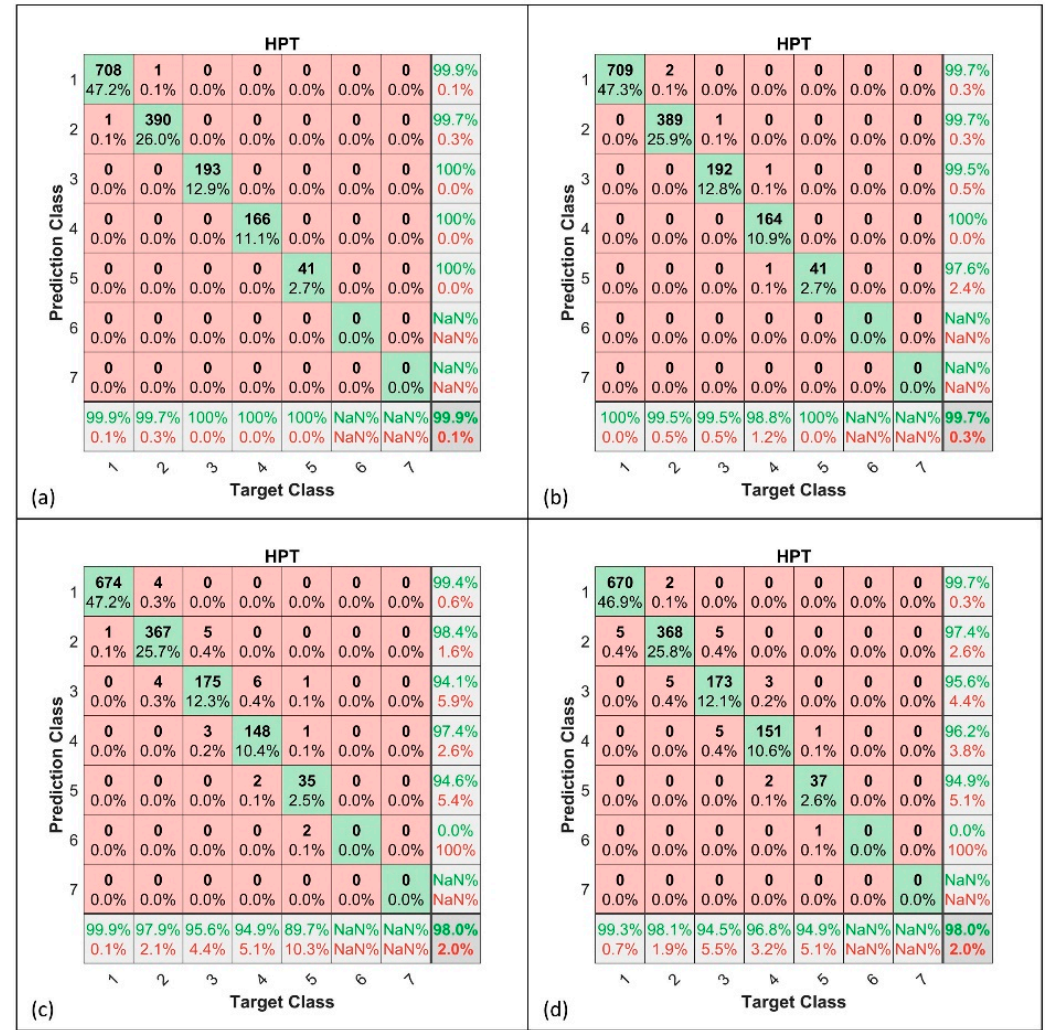

Figure 12. Confusion matrix for HPT. (a): Single-component FFNN; (b): single-component KPCA + FFNN; (c): multi-component FFNN; (d): multi-component KPCA + FFNN. 


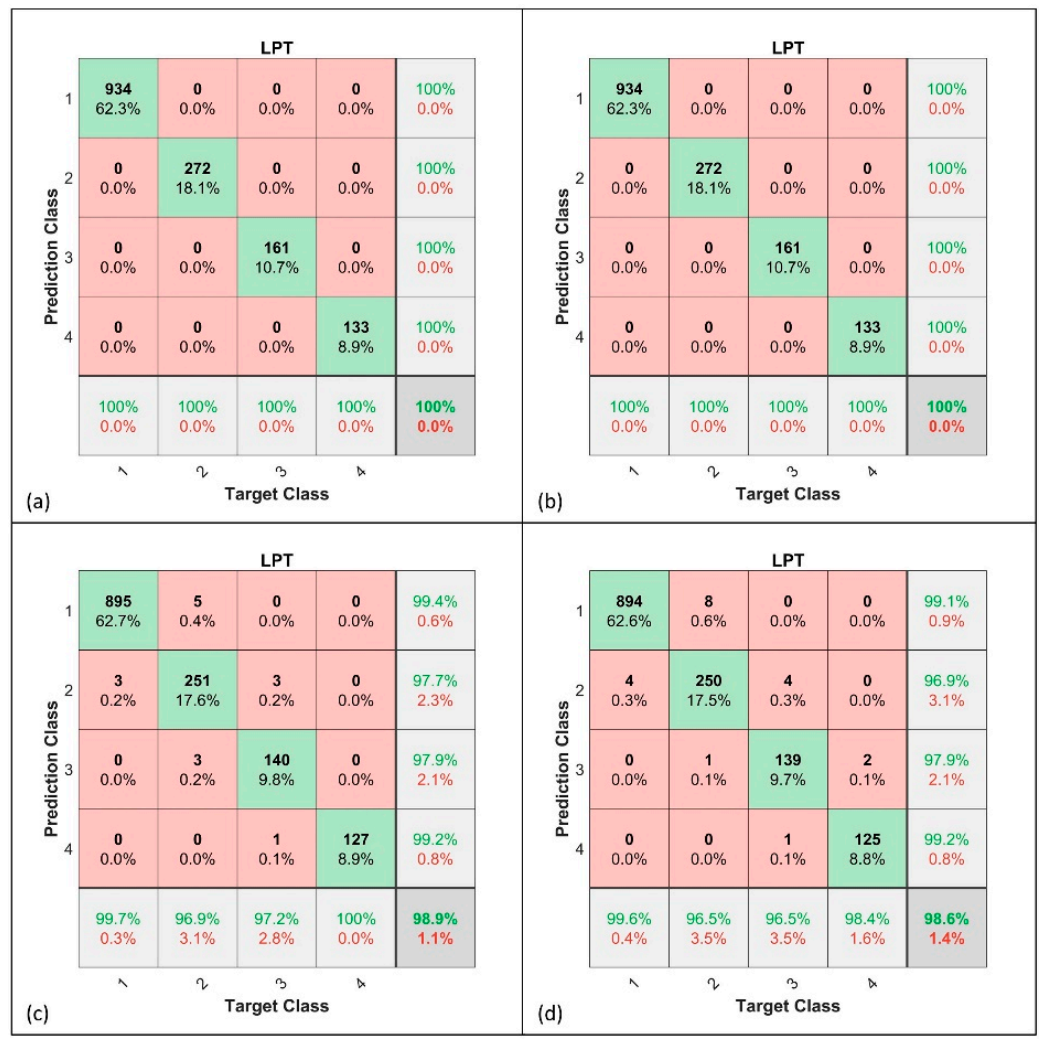

Figure 13. Confusion matrix for LPT. (a): Single-component FFNN; (b): single-component KPCA + FFNN; (c): multi-component FFNN; (d): multi-component KPCA + FFNN.

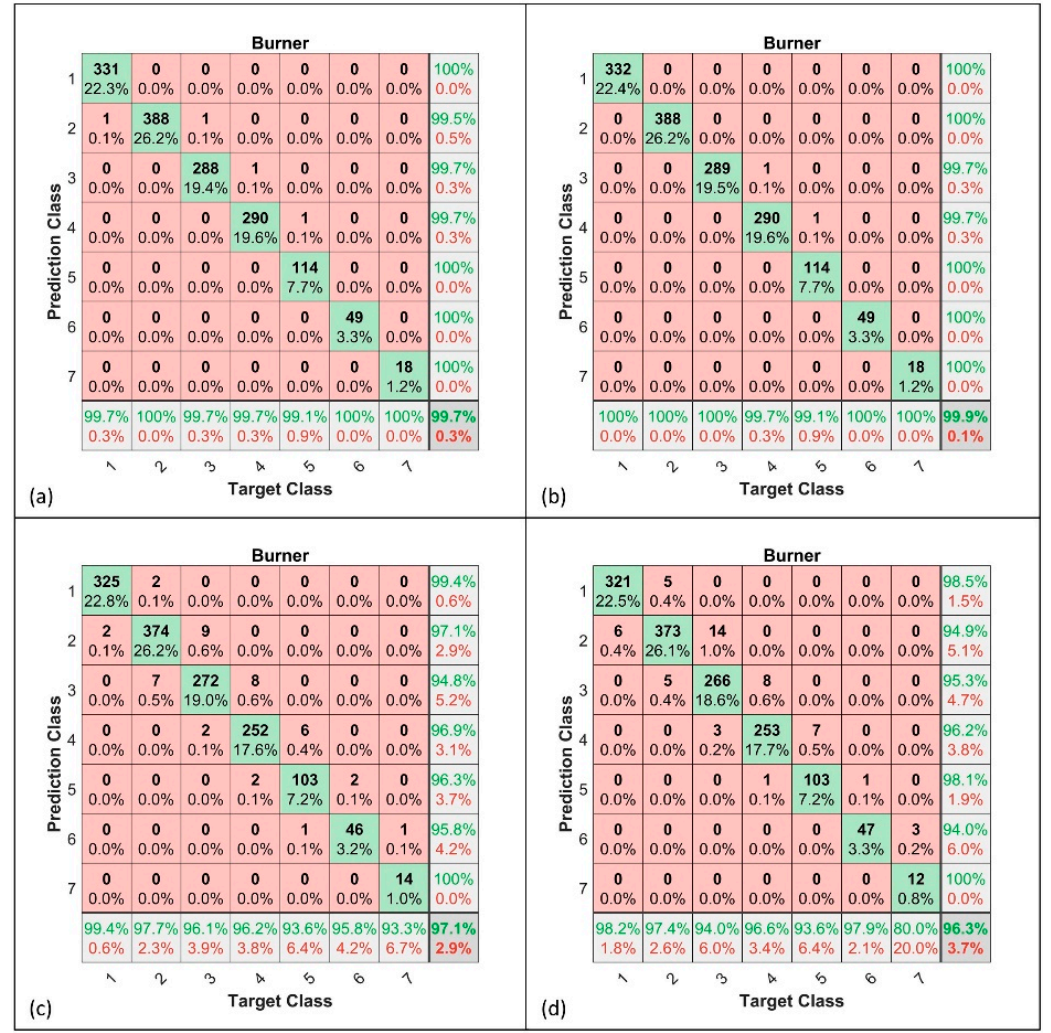

Figure 14. Confusion matrix for burner. (a): Single-component FFNN; (b): single-component KPCA + FFNN; (c): multi-component FFNN; (d): multi-component KPCA + FFNN. 


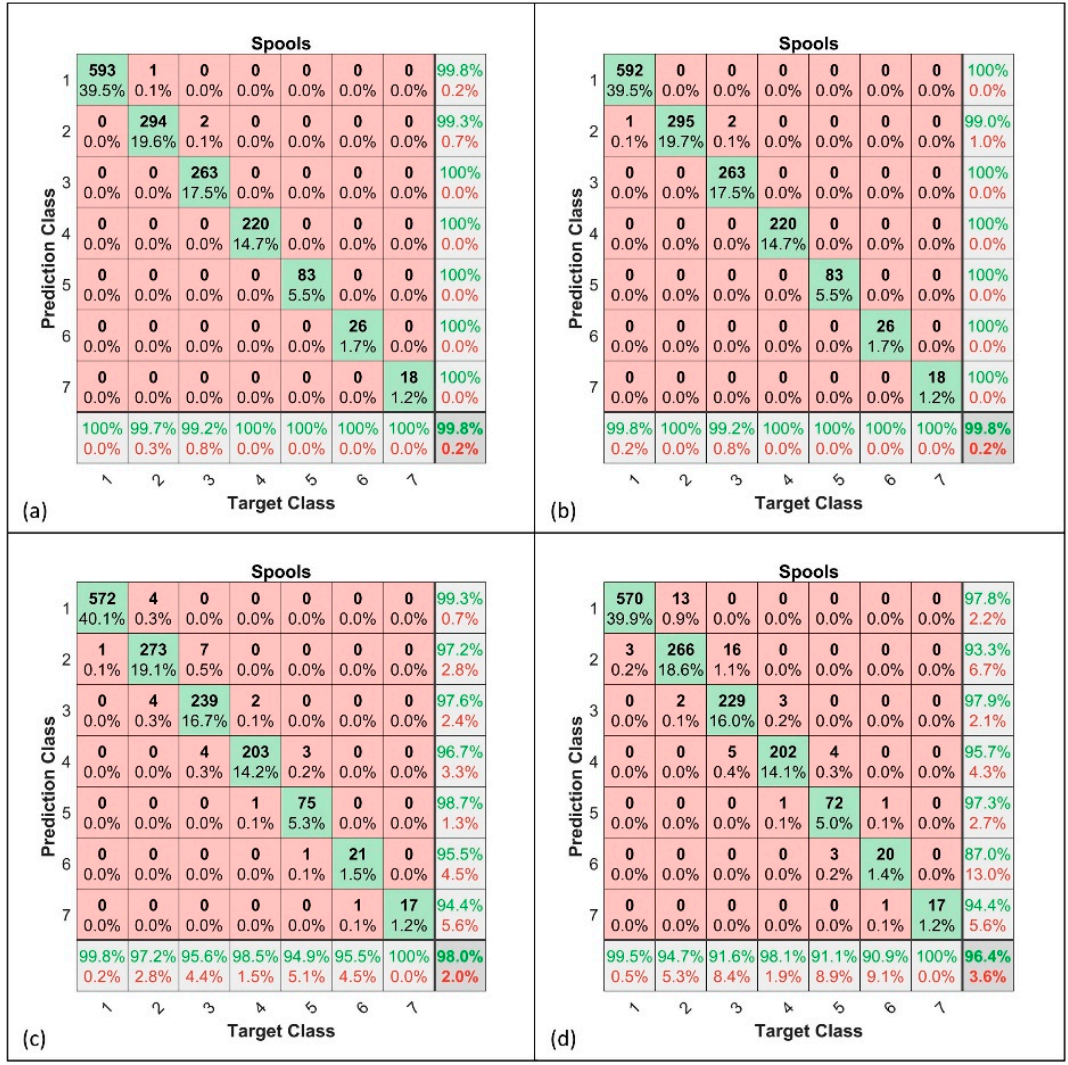

Figure 15. Confusion matrix for shaft transmissions. (a): Single-component FFNN; (b): singlecomponent KPCA + FFNN; (c): multi-component FFNN; (d): multi-component KPCA + FFNN.

\section{Conclusions}

The goal of the study presented here was to obtain a valid EHM system capable of predicting the so-called Performance Parameters of some of the principal components constituting a turboshaft, i.e., compressor, high-pressure turbine, low-pressure turbine, burner, and shafts. Artificial intelligence has been used to reach the target. FFNNs were exploited and trained to predict the values of the PPs starting from the values of flight mission data and some state engine variables, which could be measured in a real engine by installed sensors. The input parameters included the flight Mach number, ambient total pressure and temperature, total pressures and total temperatures in various engine stations, high-pressure spool speed, low-pressure spool torque, overall pressure ratio and fuel flow-PLA ratio. In the present work, the datasets used to train neural networks were obtained from a series of steady-state GSP simulations, implementing the six different analyzed degradation cases. Each simulation was characterized by a different degradation level and condition. The degradation was implemented by changing the values of the PPs in the GSP block model of the simulated engine. The degraded values of the PPs were obtained using an adequate equation useful for obtaining a random value between a maximum and a minimum. The maximum and minimum acceptable values of the PPs were dictated by the considered range of percentage variation with respect to the healthy condition. The prediction of PPs by means of neural networks was performed in two ways; first by training the networks with the original datasets (as obtained from the simulations) and next by training the networks with the reduced datasets (obtained applying KPCA to the original datasets). The results were compared. The prediction of PPs in the case without the use of KPCA produced very good results, both for single-component degradation cases and for multi-component degradation cases. Application of the KPCA technique does not lead to a serious lowering of the reliability, both in the case of single-component and multi-component degradation. Finally, based on values of the PPs, a degraded component 
class was associated with each observation point in the datasets, both for the datasets obtained from the GSP simulations and for the one obtained from networks prediction. The results were shown using the confusion matrices. A data reduction technique is a valid method to reduce the number of computational efforts required, but if the use of these techniques compromises the efficiency of the system, it may be preferable not to use it. However, multiple single-output ANNs would be implemented to improve the prediction of multi-component degradation predictions; in this case, the use of KPCA will be useful to reduce the time of computations and should not impact the prediction performance, as shown in the single-component degradation case that presents few outputs (one or two) for each condition to be predicted. In the future, we will evaluate the robustness of the method in the presence of measurement uncertainty via input data corrupted by Gaussian noise.

Author Contributions: Conceptualization, M.G.D.G.; methodology, M.G.D.G. and L.S.; software, N.M. and L.S.; validation, N.M.; formal analysis, N.M.; investigation, N.M. and L.S.; resources, A.F.; data curation, L.S.; writing-original draft preparation, N.M. and M.G.D.G.; writing-review and editing, M.G.D.G.; supervision, M.G.D.G. and A.F.; project administration, A.F.; funding acquisition, A.F. All authors have read and agreed to the published version of the manuscript.

Funding: This research was funded by the Italian Ministry of University and Research, project PON "SMEA", code PON03PE_00067_5.
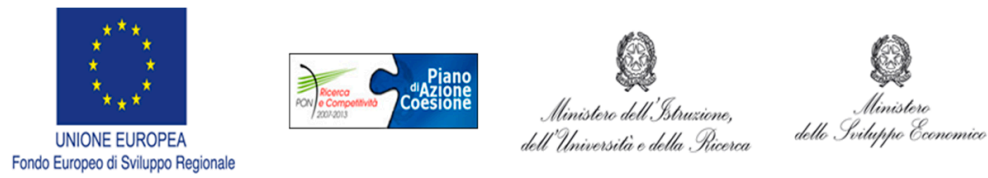

2. Ministro per la Coesione Territoriale

Institutional Review Board Statement: Not applicable.

Informed Consent Statement: Not applicable.

Conflicts of Interest: The authors declare no conflict of interest.

\section{References}

1. Vujić, D. Diagnostic systems in aircraft engines maintenance. Sci. Technol. Rev. 2005, 55, 30-34.

2. Kurz, R.; Brun, K. Gas Turbine Tutorial-Maintenance and Operating Practices Effects on Degradation and Life. In Proceedings of the 36th Turbomachinery Symposium, Texas A\&M University, Turbomachinery Laboratories, Houston, TX, USA, 11-13 September 2007.

3. Fentaye, A.D.; Baheta, A.T.; Gilani, S.I.; Kyprianidis, K.G. A Review on Gas Turbine Gas-Path Diagnostics: State-of-the-Art Methods, Challenges and Opportunities. Aerospace 2019, 6, 83. [CrossRef]

4. Meher-Homji, C.B.; Chaker, M.; Bromley, A.F. The Fouling of Axial Flow Compressors: Causes, Effects, Susceptibility, and Sensitivity. Turbo Expo Power Land Sea Air 2009, 48852, 571-590.

5. Qingcai, Y.; Li, S.; Cao, Y.; Zhao, N. Full and Part-Load Performance Deterioration Analysis of Industrial Three-Shaft Gas Turbine Based on Genetic Algorithm. Turbo Expo Power Land Sea Air 2016, 49828, V006T05A016.

6. Diakunchak, I.S. Performance Deterioration in Industrial Gas Turbines. J. Eng. Gas Turbines Power 1992, 114, 161-168. [CrossRef]

7. Swadźba, L.; Formanek, B.; Gabriel, H.M.; Liberski, P.; Podolski, P. Erosion- and corrosion-resistant coatings for aircraft compressor blades. Surf. Coat. Technol. 1993, 62, 486-492. [CrossRef]

8. Powrie, H.; Fisher, C.E. Monitoring of Foreign Objects Ingested into the Intake of a Jet Engine. In International Conference on Condition Monitoring Proceedings; University of Wales: Swansea, UK, 1999; pp. 175-190.

9. Powrie, H.; Novis, A. Gas path debris monitoring for F-35 Joint Strike Fighter propulsion system PHM. In Proceedings of the IEEE Aerospace Conference, Big Sky, MT, USA, 4-11 March 2006; p. 8.

10. DeRemer, J. Sand and Dust Erosion in Aircraft Gas Turbines. J. Am. Soc. Nav. Eng. 1950, 62, 505-511.

11. De Giorgi, M.; Campilongo, S.; Ficarella, A. Predictions of Operational Degradation of the Fan Stage of an Aircraft Engine Due to Particulate Ingestion. J. Eng. Gas Turbines Power 2015, 137, 052603. [CrossRef]

12. Ishibashi, R.; Nascimento, C., Jr. GFRBS-PHM: A genetic fuzzy rule-based system for phm with improved interpretability. In Proceedings of the 2013 IEEE Conference on Prognostics and Health Management (PHM), Gaithersburg, MD, USA, 24-27 June 2013; pp. 1-7.

13. Heimes, F.O. Recurrent neural networks for remaining useful life estimation. In Proceedings of the 2008 International Conference on Prognostics and Health Management, Denver, CO, USA, 6-9 October 2008; pp. 1-6.

14. De Giorgi, M.G.; Quarta, M. Hybrid MultiGene Genetic Programming-Artificial neural networks approach for dynamic performance prediction of an aeroengine. Aerosp. Sci. Technol. 2020, 103, 105902. [CrossRef] 
15. De Giorgi, M.G.; Strafella, L.; Ficarella, A. Neural Nonlinear Autoregressive Model with Exogenous Input (NARX) for Turboshaft Aeroengine Fuel Control Unit Model. Aerospace 2021, 8, 206. [CrossRef]

16. Palme, T.; Breuhaus, P.; Assadi, M.; Klein, A.; Kim, M. Early Warning of Gas Turbine Failure by Nonlinear Feature Extraction Using an Auto-Associative Neural Network Approach. Turbo Expo Power Land Sea Air 2011, 54631, $293-304$.

17. Alozie, O.; Li, Y.G.; Pilidis, P.; Korakianitis, T.; Ren, W.; Shong, X.; Wu, X. An Integrated Principal Component Analysis, Artificial Neural Network and Gas Path Analysis Approach for Multi-Component Fault Diagnostics of Gas Turbine Engines. Turbo Expo Power Land Sea Air 2020, 84140, V005T05A023.

18. De Giorgi, M.G.; Ficarella, A.; De Carlo, L. Jet engine degradation prognostic using artificial neural networks. Aircr. Eng. Aerosp. Technol. 2019, 92, 296-303. [CrossRef]

19. De Giorgi, M.G.; Campilongo, S.; Ficarella, A. Development of a real time intelligent health monitoring platform for aero-engine. MATEC Web Conf. 2018, 233, 00007. [CrossRef]

20. GSP 11 User Manual. Available online: https://www.gspteam.com/GSPsupport/OnlineHelp/index.html?documentation.htm (accessed on 12 January 2022).

21. Donateo, T.; De Pascalis, C.L.; Strafella, L.; Ficarella, A. Optimal Energy Management of a Hybrid Electric Helicopter for Urban Air-Mobility. IOP Conf. Ser. Mater. Sci. Eng. 2021, 1024, 012074. [CrossRef]

22. Jolliffe, I.T. Principal Component Analysis; Springer: New York, NY, USA, 1986.

23. Kim, K.I.; Jung, K.; Kim, H.J. Face recognition using kernel principal component analysis. IEEE Signal Process. Lett. 2002, 9, 40-42. 